\title{
BMJ Open Effectiveness of a multidisciplinary critical pathway based on a computerised physician order entry system for ST-segment elevation myocardial infarction management in the emergency department: a retrospective observational study
}

\author{
Yoo Seok Park, ${ }^{1}$ Sung Phil Chung, ${ }^{1}$ Je Sung You, ${ }^{1}$ Min Joung Kim, ${ }^{1}$ \\ Hyun Soo Chung, ${ }^{1}$ Jung Hwa Hong, ${ }^{2}$ Hye Sun Lee, ${ }^{2}$ Jinwon Wang, ${ }^{1}$ Incheol Park ${ }^{1}$
}

To cite: Park YS, Chung SP, You JS, et al. Effectiveness of a multidisciplinary critical pathway based on a computerised physician order entry system for ST-segment elevation myocardial infarction management in the emergency department: a retrospective observational study. BMJ Open 2016;6: e011429. doi:10.1136/ bmjopen-2016-011429

- Prepublication history for this paper is available online. To view these files please visit the journal online (http://dx.doi.org/10.1136/ bmjopen-2016-011429).

Received 9 February 2016 Revised 17 June 2016 Accepted 21 July 2016

CrossMark

For numbered affiliations see end of article.

Correspondence to Dr Je Sung You; youjsmd@yuhs.ac

\section{ABSTRACT}

Objectives: The purpose of this study was to investigate whether a multidisciplinary organised critical pathway (CP) for ST-segment elevation myocardial infarction (STEMI) management can significantly attenuate differences in the duration from emergency department (ED) arrival to evaluation and treatment, regardless of the arrival time, by eliminating off-hour and weekend effects.

Design: Retrospective observational cohort study.

Setting: 2 tertiary academic hospitals.

Participants: Consecutive patients in the Fast Interrogation Rule for STEMI (FIRST) program.

Interventions: A study was conducted on patients in the FIRST program, which uses a computerised physician order entry (CPOE) system. The patient demographics, time intervals and clinical outcomes were analysed based on the arrival time at the ED: group 1, normal working hours on weekdays; group 2, off-hours on weekdays; group 3, normal working hours on weekends; and group 4, off-hours on weekends.

\section{Primary and secondary outcome measures:}

Clinical outcomes categorised according to 30-day mortality, in-hospital mortality and the length of stay.

Results: The duration from door-to-data or FIRST activation did not differ significantly among the 4 groups. The median duration between arrival and balloon placement during percutaneous coronary intervention did not significantly exceed $90 \mathrm{~min}$, and the proportions (89.6-95.1\%) of patients with door-toballoon times within 90 min did not significantly differ among the 4 groups, regardless of the ED arrival time $(p=0.147)$. Moreover, no differences in the 30 -day $(p=0.8173)$ and in-hospital mortality $(p=0.9107)$ were observed in patients with STEMI.

Conclusions: A multidisciplinary CP for STEMI based on a CPOE system can effectively decrease disparities in the door-to-data duration and proportions of

\section{Strengths and limitations of this study}

- In the present study, the Fast Interrogation Rule for ST-segment elevation myocardial infarction (STEMI) (FIRST) program, based on a computerised physician order entry and an automated short message service, was used to operate an effective pre-existing FIRST team for rapid STEMI management in our two affiliated hospitals.

- A multidisciplinary critical pathway (CP) for STEMI was found to effectively decrease disparities in the door-to-data interval and the proportions of patients with door-to-balloon times within $90 \mathrm{~min}$, regardless of the emergency department arrival time.

- The application of a multidisciplinary CP could also help attenuate off-hour and weekend effects in terms of the clinical outcomes after STEMI.

- However, owing to the retrospective observational nature of the study, it was impossible to perform a direct internal comparison with clinical data obtained before the implementation, because it was difficult to select a cohort with similar inclusion criteria as that of the cohort in this study.

patients with door-to-balloon times within $90 \mathrm{~min}$, regardless of the ED arrival time. The application of a multidisciplinary CP may also help attenuate off-hour and weekend effects in STEMI clinical outcomes.

\section{INTRODUCTION}

Acute myocardial infarction (AMI), defined as complete occlusion of the epicardial 
coronary artery, is a leading cause of disability and mortality worldwide. ${ }^{1-3}$ Approximately $15 \%$ of those who experience myocardial infarction (MI) reportedly die. ${ }^{4}$ The primary goal in the treatment of AMI is therefore early reperfusion therapy through intravenous administration of tissue plasminogen activator (intravenous-tPA) or through emergency cardiac procedures such as a primary percutaneous coronary intervention (PCI). ${ }^{35} 6$ Ideally, primary PCI should be conducted with a door-to-balloon time within $90 \mathrm{~min}$ in patients with STEMI. ${ }^{7}$ A delay of $\sim 30$ min in the door-to-balloon time has been reported to increase in-hospital mortality by $20-30 \%$ in patients with STEMI, regardless of door-to-balloon times of up to $180 \mathrm{~min} .{ }^{2}$ However, there is often limited staff availability during off-hours admissions as compared to admissions during regular working hours. ${ }^{8}$ Previous studies have shown increased mortality in different conditions such as cancer, aortic aneurysm, stroke, duodenal ulcer, epiglottitis and pulmonary embolism, among others, when patients are admitted during off-hours or on weekends. ${ }^{9}{ }^{10}$ This phenomenon is commonly referred to as the 'weekend effect' or 'off-hour effect'. ${ }^{11}$ Although off-hour and weekend effects on mortality in patients with AMI are controversial, recent studies have suggested that patients with AMI admitted during off-hours and on weekends show delayed door-to-balloon times and higher short-term mortality. ${ }^{2}$ However, it may be difficult to continuously maintain specialised and standardised care, which in turn may result in disparities in critical factors in STEMI management, such as delays in the acquisitions of ECG and blood tests, and the use of thrombolytic treatment, according to the emergency department (ED) arrival time. At our institutions, the applications of computerised physician order entry (CPOE)-based multidisciplinary CPs for ischaemic stroke and postpartum haemorrhage have demonstrated attenuations in the durations of presentation to therapy. ${ }^{12}{ }^{13}$ In our hospitals, considering the low level of awareness of the public and emergency medical services (EMS) in Korea regarding the importance of rapid diagnosis and treatment of acute coronary syndrome, the Fast Interrogation Rule for STEMI (FIRST) program, which is based on a CPOE system, has been used to operate an effective pre-existing FIRST team for rapid STEMI management since 2007. The purpose of this study was to investigate whether an organised critical pathway (CP) for the management of STEMI can effectively attenuate the differences in the duration from ED arrival to evaluation and treatment, regardless of the time of arrival at the ED, by eliminating the off-hour and weekend effects.

\section{MATERIALS AND METHODS}

A retrospective, observational cohort study for all consecutive patients included in the FIRST program was conducted at two tertiary academic hospitals, Yonsei University College of Medicine-affiliated Severance and
Gangnam Severance Hospitals, with annual ED censuses of 65000 and 85000 , respectively, during the study period. In 2007, we implemented a multidisciplinary CP based on a CPOE system, known as FIRST, at the two hospitals. Our CP for AMI management was designed to reduce unnecessary in-hospital time delays through a CPOE-based alert system, short message service (SMS) and simple standing orders through the activation stage. The study included all patients admitted between 1 January 2010 and 30 December 2012 with STEMI who presented to the ED within 12 hours of the onset of chest pain. The CPOE system was used for program activation and deactivation, communication and consultation, entering predetermined standing order sets, providing specific protocols and current guidelines, and to assess program efficacy. ${ }^{4}$ On arrival of a patient at the ED, the physicians, nurses and emergency medical technicians in the triage area identified candidates for the FIRST program as soon as possible according to predetermined protocols. Preferentially, patients with typical or non-specific suspected symptoms of AMI (chest pain, epigastric pain, syncope, dizziness, vertigo, shock, dyspnoea, nausea and/or vomiting) were examined simultaneously as a 12-lead ECG is performed in the triage area. The guidelines included criteria for CP activation based on the ECG criteria in the standard STEMI guidelines and the duration after the onset of chest pain. The ST-segment elevations in the ECG criteria were defined as (1) a J-point elevation on two or more contiguous leads, with a threshold of more than $2 \mathrm{~mm}$ in the precordial leads or more than $1 \mathrm{~mm}$ in other leads and (2) a new or presumed new left bundle branch block. When a patient had at least one ECG warning criterion by predetermined ST-segment elevations on ED arrival within 12 hours of the onset of symptoms, an ED physician in the triage area activated the FIRST program by selecting the activation icon on the order entry window. Once the program is activated, the name of the patient is highlighted in bright yellow in the patient list; thereby, each team member can easily and immediately recognise the patients in the FIRST program. $^{4}{ }^{9}$ The mobile phone number of each member of the FIRST team has been previously registered in a computerised order-entry database for physicians, and a program that links consultation orders to an automated SMS has been developed. The SMS text is immediately transmitted to the team members after consultation. At the same time, an ED physician immediately consults with an on-call cardiologist or with coronary catheterisation staff and a senior ED physician. Medical orders for blood tests are automatically displayed, and an alarm with a beeping sound and pop-up window is added to the technician's monitor. ${ }^{49}$ Thus, at the same time that medical orders are submitted, laboratory technicians are alerted and can prepare for the arrival of blood samples. The on-call cardiologist and coronary catheterisation staff are also alerted and can prepare for the arrival of the patient. These preparations 
enable immediate and rapid examinations. All members can indirectly identify SMS reception using the CPOE system and directly by using their mobile phone. After activation of the FIRST CP, predetermined orders are also activated using CPOE. All members who receive an SMS should change the status of the charging order using CPOE from 'order' to 'registration'. In addition, all members of the CP can share ECG data and information of patients in real time using the CPOE system, including electronic medical records. The on-call cardiologists first assess the ECG for STEMI, symptoms characteristics, medical history of the patient and risk stratification in the ED. Subsequently, they determine whether emergent thrombolytic treatment is indicated or whether PCI is considered as a delayed procedure. Furthermore, they decide whether intravenous t-PA or primary PCI is suitable for the treatment. Finally, they decide to deactivate the FIRST program using CPOE. In this study, to compare time-related factors among the groups, patients were excluded from the study if return of spontaneous circulation occurred after out-of-hospital cardiac arrest or if ST-segment elevation was revealed only on follow-up ECG in patients who were included in the $\mathrm{CP}$, because these factors increase the door-toballoon time. Patients with ST-segment elevation revealed only on follow-up ECG were defined as those who were admitted to the ED within 12 hours of the onset of chest pain but did not reveal the ST-segment elevations of the ECG criteria on the initial ECG in the ED. Owing to ongoing chest pain, these patients were followed up by ECG during their ED stay and often revealed ST-segment elevations on the follow-up ECG. The patients' demographics, Killip class, PCI lesions and laboratory tests for cardiac enzymes were examined. The time intervals assessed included the door-to-ECG, ECG-to-FIRST activation, FIRST-to-PCI or t-PA and door-to-PCI times. The patients were classified based on the arrival time at the ED. Patients who arrived between 9:00 and 18:00 and between 18:00 and 9:00 were arbitrarily designated as having arrived during working hours and off-hours, respectively. The weekend was defined as the period from Saturday to Sunday. National holidays were also included in the weekend classification. The patients were divided into four groups according to their arrival time: group 1 (patients who arrived during working hours on a weekday), group 2 (patients who arrived during off-hours on a weekday), group 3 (patients who arrived during working hours on a weekend) and group 4 (patients who arrived during offhours on a weekend). ${ }^{10}$ The clinical outcomes were categorised and examined according to the 30-day mortality, overall in-hospital mortality and single length of stay (LOS), and compared between the four groups.

\section{Statistics}

Demographic and clinical data are presented as the median (minimum, maximum), frequency or mean \pm SD as appropriate. Differences in the demographic characteristics, time intervals, mortality and LOS among the four groups were compared with the Kruskal-Wallis test for continuous variables and the $\chi^{2}$ or Fisher's exact test for categorical variables. Post hoc analysis was performed with the Dunn procedure for continuous variables and the $\chi^{2}$ or Fisher's exact test for two pairs of categorical variables. Univariate Cox regression was performed to identify the effects on mortality of the door-to-PCI and FIRST activation-to-PCI intervals. Survival curves were obtained using the Kaplan-Meier analysis, and the log-rank test was performed for survival comparisons among the four groups. All statistical analyses were conducted with SAS V.9.2 (SAS Institute, Cary, North Carolina, USA) and $\mathrm{p}<0.05$ were considered significant.

\section{RESULTS}

At the two affiliated hospitals, there were 1043 consecutive patients admitted to the EDs included in the FIRST CP. Among 729 (69.9\%) patients who underwent emergency coronary angiography, $560(53.7 \%)$ were diagnosed with STEMI. However, 82 patients were excluded from the analysis because of time delay factors such as out-of-hospital cardiac arrest $(n=23)$, ST-segment elevation on follow-up ECG only ( $\mathrm{n}=24)$, delayed PCI because of a $Q$ wave without symptoms $(n=22)$, and 'do not attempt resuscitation' status $(n=3)$. Thus, a total of 488 patients with STEMI were finally enrolled in this study. There were no significant differences, except in the family history for coronary artery disease and weight, among the demographic characteristics of the four groups (table 1). The time interval between arrival at the ED and ECG or the activation of FIRST did not differ significantly among the four groups (table 2). However, the time intervals from FIRST activation-to-PCI and door-to-PCI showed significant differences among the four groups. The door-to-balloon duration in patients with STEMI should be within $90 \mathrm{~min}$. Among patients who underwent PCI in the four groups, the median duration between arrival at the ED and PCI balloon placement did not significantly exceed $90 \mathrm{~min}$ (table 3). The proportions of patients with door-to-balloon times within 90 min ranged between $89.6 \%$ and $95.1 \%$ in the four groups (figure 1 ) and did not reveal any significant differences $(\mathrm{p}=0.147)$. Univariate cox regression was performed to identify the effects on mortality according to the durations of door-to-PCI balloon placement and the activation of FIRST-to-PCI balloon placement; these did not affect in-hospital or 30-day mortality, regardless of the ED arrival time (table 3 ).

The survival curves for in-hospital mortality $(\mathrm{p}=0.8668)$ and 30-day mortality $(\mathrm{p}=0.8963)$ did not differ significantly among all patients, regardless of the ED arrival time (figure 2A, B). The in-hospital and 30-day mortality rates during a single hospital stay were $7.99 \%$ and $8.4 \%$, respectively, among patients with 
Table 1 Clinicodemographic characteristics of all patients

\begin{tabular}{|c|c|c|c|c|c|c|c|c|c|c|c|}
\hline \multirow[b]{2}{*}{ Characteristics } & \multicolumn{4}{|c|}{ Median (minimum-maximum) } & \multirow[b]{2}{*}{ Overall } & \multicolumn{6}{|c|}{ Post hoc analysis $p$ value } \\
\hline & (group 1) $(n=206)$ & (group 2) $(n=154)$ & (group 3) $(n=61)$ & (group 4) $(n=67)$ & & $\begin{array}{l}1) \text { vs } \\
(2)\end{array}$ & $\begin{array}{l}\text { (1) vs } \\
\text { (3) }\end{array}$ & $\begin{array}{l}\text { (1) vs } \\
(4)\end{array}$ & $\begin{array}{l}\text { (2) vs } \\
\text { (3) }\end{array}$ & $\begin{array}{l}\text { (2) vs } \\
\text { (4) }\end{array}$ & $\begin{array}{l}\text { (3) vs } \\
\text { (4) }\end{array}$ \\
\hline Age (years) & $64(32-98)$ & $62(29-91)$ & $63(41-91)$ & $63(28-92)$ & 0.8381 & & & & & & \\
\hline Sex & & & & & 0.2205 & & & & & & \\
\hline Male & $162(78.64)$ & $131(85.06)$ & 45 (73.77) & $52(77.61)$ & & & & & & & \\
\hline Female & $44(21.36)$ & $23(14.94)$ & $16(26.23)$ & 15 (22.39) & & & & & & & \\
\hline Systolic BP (mm Hg) & $125(43-223)$ & $131(59-268)$ & $130(68-220)$ & $133(65-202)$ & 0.4672 & & & & & & \\
\hline Diastolic BP $(\mathrm{mm} \mathrm{Hg})$ & $79.5(9-133)$ & $82.5(43-184)$ & $82(40-122)$ & $80(43-125)$ & 0.1289 & & & & & & \\
\hline Pulse rate (bpm) & $79.5(34-150)$ & $80(35-145)$ & $82(43-134)$ & $81(34-156)$ & 0.7674 & & & & & & \\
\hline Diabetic mellitus & $67(32.52)$ & $48(31.17)$ & $21(34.43)$ & $25(37.31)$ & 0.8251 & & & & & & \\
\hline Hypertension & $115(55.83)$ & $75(48.7)$ & $26(42.62)$ & $41(61.19)$ & 0.1028 & & & & & & \\
\hline Chronic kidney disease & $11(5.34)$ & $8(5.19)$ & $1(1.64)$ & $4(5.97)$ & 0.666 & & & & & & \\
\hline Stroke & $12(5.83)$ & $11(7.14)$ & $2(3.28)$ & $3(4.48)$ & 0.7574 & & & & & & \\
\hline Variant angina & $3(1.46)$ & $1(0.65)$ & $1(1.64)$ & $1(1.49)$ & 0.8251 & & & & & & \\
\hline Stable angina & 5 (2.43) & $4(2.6)$ & 2 (3.28) & $2(2.99)$ & 0.9306 & & & & & & \\
\hline Myocardial infarction & $12(5.83)$ & $12(7.79)$ & $1(1.64)$ & $4(5.97)$ & 0.4066 & & & & & & \\
\hline Heart failure & $3(1.46)$ & $3(1.95)$ & $1(1.64)$ & $1(1.49)$ & 0.9534 & & & & & & \\
\hline S/P PTCA & $17(8.25)$ & 19 (12.34) & $4(6.56)$ & $10(14.93)$ & 0.2487 & & & & & & \\
\hline S/P CABG & $1(0.49)$ & $1(0.65)$ & $1(1.64)$ & $1(1.49)$ & 0.4873 & & & & & & \\
\hline Current smoker & 81 (39.32) & $74(48.05)$ & 20 (32.79) & $35(52.24)$ & 0.0535 & & & & & & \\
\hline Hypercholesterolaemia & $17(8.25)$ & $11(7.14)$ & $3(4.92)$ & $6(8.96)$ & 0.8339 & & & & & & \\
\hline Family history of CAD & & & & & $0.0122^{*}$ & 0.0096 & 0.5918 & 0.6816 & 0.0217 & 0.2422 & 0.4972 \\
\hline No & $201(97.57)$ & $141(91.56)$ & $61(100)$ & $65(97.01)$ & & & & & & & \\
\hline Yes & $5(2.43)$ & $13(8.44)$ & $0(0)$ & $2(2.99)$ & & & & & & & \\
\hline Height $(\mathrm{cm})$ & $167(140-186)$ & $168(140-183)$ & $167(146-178)$ & $168(140-190)$ & 0.4864 & & & & & & \\
\hline Weight (kg) & $65(43-96)$ & $69.3(37.5-120)$ & $65.3(17.2-86)$ & $68.3(36.1-133)$ & $0.0417^{*}$ & 0.0334 & 0.3858 & 0.1586 & 0.0189 & 0.8591 & 0.0657 \\
\hline Creatine kinase & $143.5(20-5514)$ & $127(14-10560)$ & $122(53-8400)$ & $110(25-2132)$ & 0.222 & & & & & & \\
\hline Creatine kinase-MB & $4.43(0.56-619.4)$ & $3.32(0.7-638)$ & $5.29(0.92-600)$ & $3.5(0.76-172.8)$ & 0.2161 & & & & & & \\
\hline Troponin-T & $0.06(0-10)$ & $0.03(0-9.86)$ & $0.06(0-10)$ & $0.04(0-18.22)$ & 0.0959 & & & & & & \\
\hline Mode of transport & & & & & 0.3446 & & & & & & \\
\hline $\begin{array}{l}\text { Emergency medical } \\
\text { service }\end{array}$ & $105(50.97)$ & $88(57.14)$ & 38 (63.33) & $37(55.22)$ & & & & & & & \\
\hline Private vehicles & $101(49.03)$ & $66(42.86)$ & $22(36.67)$ & $30(44.78)$ & & & & & & & \\
\hline Killip class & & & & & 0.3609 & & & & & & \\
\hline 1 & $125(60.68)$ & $104(67.53)$ & $34(56.67)$ & $42(62.69)$ & & & & & & & \\
\hline II & 28 (13.59) & $13(8.44)$ & 8 (13.33) & $13(19.4)$ & & & & & & & \\
\hline III & $23(11.17)$ & 20 (12.99) & 7 (11.67) & 7 (10.45) & & & & & & & \\
\hline IV & $30(14.56)$ & $17(11.04)$ & $11(18.33)$ & $5(7.46)$ & & & & & & & \\
\hline CPR in ED & & & & & 0.1134 & & & & & & \\
\hline No & $201(97.57)$ & $154(100)$ & 59 (96.72) & $66(98.51)$ & & & & & & & \\
\hline Yes & $5(2.43)$ & $0(0)$ & 2 (3.28) & $1(1.49)$ & & & & & & & \\
\hline
\end{tabular}

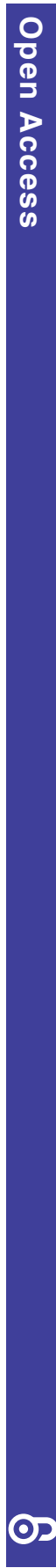




\section{Characteristics}

(group 1) (n=206)

(group 2) $(n=154)$

(group 3) $(n=61)$

(group 4) (n=67)

Post hoc analysis $p$ value

\begin{tabular}{lcccc}
\hline Chest pain & & & \\
$\quad$ No & $23(11.17)$ & $13(8.44)$ & $5(8.2)$ & $6(8.96)$ \\
Yes & $183(88.83)$ & $141(91.56)$ & $56(91.8)$ & $61(91.04)$ \\
PCl lesion & & & \\
LAD & $94(46.08)$ & $65(42.76)$ & $39(65)$ & $31(46.27)$ \\
RCA & $77(37.75)$ & $52(34.21)$ & $15(25)$ & $24(35.82)$ \\
LCX & $9(4.41)$ & $12(7.89)$ & $1(1.67)$ & $4(5.97)$ \\
More than two & $24(11.76)$ & $23(15.13)$ & $5(8.33)$ & $8(11.94)$
\end{tabular}

(1) vs (1) vs (1) vs

$\begin{array}{lll} & \\ \text { 2) vs } & \text { (2) vs (3) vs }\end{array}$

vessels

The patients were divided into four groups: group 1 (patients who arrived during working hours on a weekday), group 2 (patients who arrived during off-hours on a weekday), group 3 (patients who arrived during working hours on a weekend) and group 4 (patients who arrived during off-hours on a weekend).

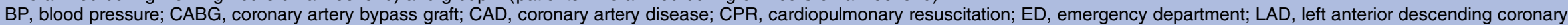
artery; LCX, left circumflex coronary artery; PCl, percutaneous coronary intervention; PTCA, percutaneous transluminal coronary angioplasty; RCA, Right coronary artery.

${ }^{*} \mathrm{p}<0.05$

Table 2 Time intervals and clinical outcomes of all patients

\begin{tabular}{|c|c|c|c|c|c|c|c|c|c|c|c|}
\hline & \multicolumn{4}{|c|}{ Median (minimum-maximum) } & \multirow{2}{*}{$\begin{array}{l}\text { Overall } \\
\text { p Value }\end{array}$} & \multicolumn{6}{|c|}{ Post hoc analysis $p$ value } \\
\hline & (group 1) $(n=206)$ & (group 2) $(n=154)$ & (group 3) $(n=61)$ & (group 4) $(n=67)$ & & (1) vs (2) & (1) vs (3) & (1) vs (4) & (2) vs (3) & (2) vs (4) & (3) vs (4) \\
\hline \multicolumn{12}{|l|}{ Time outcomes } \\
\hline Door-to-ECG (min) & $3(0-64)$ & $2(0-26)$ & $1(0-38)$ & $3(0-49)$ & 0.0749 & 0.1503 & 0.0648 & 0.437 & 0.4433 & 0.0728 & $0.0324^{*}$ \\
\hline ECG-to-FIRST (min) & $4(0-64)$ & $4(0-29)$ & $4(0-26)$ & $4(0-27)$ & 0.7129 & 0.6651 & 0.3966 & 0.7804 & 0.262 & 0.9623 & 0.3577 \\
\hline FIRST-to-balloon (min) & $41.5(16-210)$ & $52(22-147)$ & $53(13-96)$ & $59(25-98)$ & $<0.0001^{*}$ & $<0.0001^{*}$ & $<0.0001^{*}$ & $<0.0001^{\star}$ & 0.9656 & $0.0102^{*}$ & $0.0368^{*}$ \\
\hline Door-to-balloon (min) & $51.5(22-226)$ & $62(31-152)$ & $61(26-130)$ & $71(27-124)$ & $<0.0001^{*}$ & $<0.0001^{*}$ & $0.0024^{*}$ & $<0.0001^{\star}$ & 0.5535 & $0.0078^{*}$ & $0.0068^{*}$ \\
\hline Length of stay & $5(1-61)$ & $5(1-54)$ & $5(3-55)$ & $5(1-43)$ & 0.3127 & 0.2591 & 0.5076 & 0.0781 & 0.8758 & 0.3835 & 0.393 \\
\hline \multicolumn{12}{|l|}{ Clinical outcomes } \\
\hline In-hospital mortality (1-5) & & & & & 0.9107 & 0.5822 & 0.7922 & 0.9565 & 0.5764 & 0.6421 & 0.8644 \\
\hline No & $188(91.26)$ & $143(92.86)$ & $55(90.16)$ & $61(91.04)$ & & & & & & & \\
\hline Yes & $18(8.74)$ & $11(7.14)$ & $6(9.84)$ & $6(8.96)$ & & & & & & & \\
\hline 30-day mortality & & & & & 0.8173 & 0.4315 & 0.7922 & 0.7441 & 0.3981 & 0.7767 & 0.6323 \\
\hline No & $188(91.26)$ & $144(93.51)$ & $55(90.16)$ & $62(92.54)$ & & & & & & & \\
\hline Yes & $18(8.74)$ & $10(6.49)$ & $6(9.84)$ & $5(7.46)$ & & & & & & & \\
\hline
\end{tabular}

The patients were divided into four groups: group 1 (patients who arrived during working hours on a weekday), group 2 (patients who arrived during off-hours on a weekday), group 3 (patients who arrived during working hours on a weekend) and group 4 (patients who arrived during off-hours on a weekend).

FIRST, the Fast Interrogation Rule for STEMI program.

${ }^{*} \mathrm{p}<0.05$. 
Table 3 Effects of the door-to-balloon duration for $\mathrm{PCl}$ and the activation of FIRST-to-balloon duration for $\mathrm{PCl}$ on in-hospital and 30-day mortality with respect to the ED arrival time

\begin{tabular}{lcc}
\hline $\begin{array}{l}\text { In-hospital } \\
\text { mortality }\end{array}$ & HR $(95 \%$ Cl) & p Value \\
\hline FIRST-to-PCI (min) & $1.005(0.992$ to 1.019$)$ & 0.4522 \\
Door-to-PCI (min) & $1.000(0.987$ to 1.014$)$ & 0.9851 \\
\hline 30-day mortality & HR $(95 \%$ Cl) & p Value \\
\hline FIRST-to-PCI (min) & $1.000(0.987$ to 1.014$)$ & 0.9851 \\
Door-to-PCl (min) & $1.006(0.992$ to 1.019$)$ & 0.4122 \\
\hline FIRST, the Fast Interrogation Rule for STEMI program; PCI, \\
percutaneous coronary intervention.
\end{tabular}

STEMI. The median LOS was 5 days and the LOS did not significantly differ among the four groups ( $p=0.3127$; table 2$)$. Moreover, differences in the 30-day $(p=0.8173)$ and in-hospital mortality $(p=0.9107)$ were not observed in patients with STEMI undergoing PCI.

\section{DISCUSSION}

Recently, a systematic review by Sorita et al demonstrated that mortality in patients with STEMI who presented during off-hours was higher than in those who presented during working hours. The authors speculated that the longer door-to-balloon times during off-hours for patients with STEMI may have contributed to the higher mortality compared to patients admitted during regular hours. ${ }^{2}$ In this study, despite minor disparities in the door-to-balloon times among the four groups, all groups had a median door-to-balloon time within $90 \mathrm{~min}$. Moreover, the proportions of patients with door-to-balloon times within $90 \mathrm{~min}$

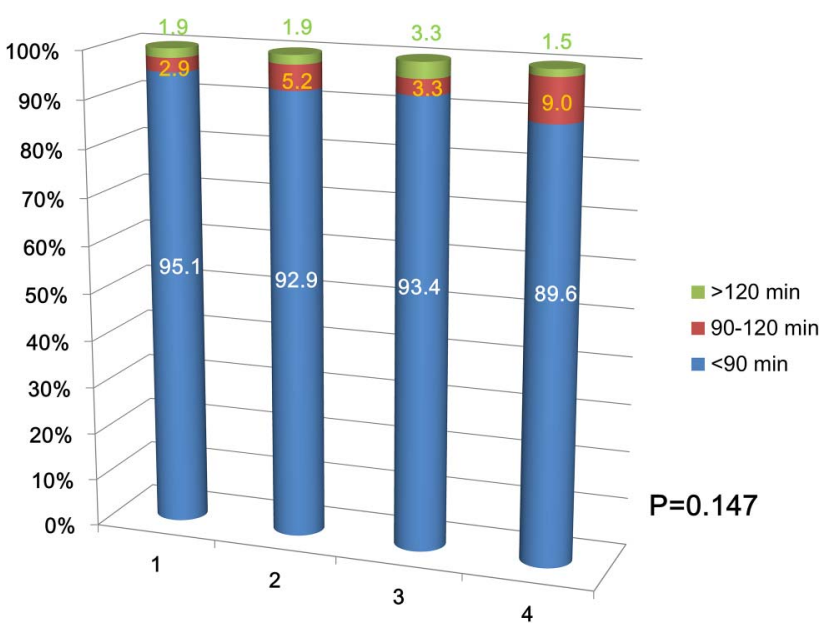

Figure 1 Proportions of patients with door-to-balloon times within 90 min with respect to the emergency department arrival time. Group 1, working hours on a weekday; group 2, off-hours on a weekday; group 3, working hours on a weekend; group 4, off-hours on a weekend. did not reveal significant differences among patients who were admitted to the ED during off-hours, regular hours, weekends and weekdays. During off-hours, the rate of PCI and differences in door-to-balloon times have been reported to most likely be associated with limited staff availability. ${ }^{2}$ However, Parikh et al reported that 'code STEMI' could decrease the median door-to-balloon duration (112 min before vs $74 \mathrm{~min}$ after code STEMI; $p<0.001$ ) and LOS ( 4 days before vs 3 days after; $p<0.01$ ) ${ }^{14}$ In one previous study, there was no significant difference in allcause mortality; ${ }^{15}$ however, a systematic review and meta-analysis revealed higher in-hospital and 30-day mortality in patients with AMI during off-hours versus those admitted during regular hours. ${ }^{2}$ The observed disparities in mortality between studies may be due to differences in the demographics of the patients with STEMI, with the mortality rates being especially high in studies conducted outside of North America, and, importantly, these rates appear to have worsened in recent years. ${ }^{2}$ Increased in-hospital mortality in AMI has been reported to be associated with off-hour admission in developing countries of EMS. For example, in Iraq, Al-Asadi et $a l^{16}$ reported that off-hour admission was significantly associated with higher mortality as compared to admission during working hours $(25.5 \%$ vs $3 \% ; \mathrm{p}<0.001)$. The delay of the time from onset of symptoms to successful PCI was closely associated with poor short-term outcomes. ${ }^{17}$ The current guidelines suggest a first medical contact-to-device time system within $90 \mathrm{~min}$ as the optimal time for treatment. ${ }^{5}{ }^{17}{ }^{18}$ Besides decreasing the door-to-balloon time, additional efforts are needed to reduce the prehospital time after symptom onset, such as increased education efforts and campaigns for the general population and EMS, coordination between the EMS and ED, performance of a 12-lead ECG by the EMS at the site of first medical contact and prehospital transmission of ECG. ${ }^{18}$ If we consider the lower levels of public awareness and education about AMI among the EMS and staff in many developing countries of EMS, implementation of an organised CP for AMI management could theoretically attenuate differences in the CP during off-hours and weekends compared to during working hours and weekdays. Herein, the FIRST CP focused on patients with suspected or distracting symptoms for AMI admitted to the ED and those who had a relatively short door-to-data (completion of an ECG) interval in the ED. Atypical symptoms are often present in patients with STEMI. Na et al showed that patients with STEMI presenting without chest pain had longer door-to-data and door-to-PCI times than patients presenting with chest pain. In fact, presentation without chest pain was an independent predictor of hospital mortality in patients with STEMI after primary PCI. ${ }^{19}$ Unfortunately, the acquisition of an ECG may be omitted in the initial assessments of these patients to the ED, and a delayed door-to-data interval may be the most critical factor for longer door-to-balloon times in patients with STEMI. Thus, standardisation of the door-to-data protocol can help improve early recognition in emergency situations. ${ }^{14}$ 
Figure 2 Kaplan-Meier survival curves with log-rank test analysis for comparisons of in-hospital (A) and 30-day (B) mortality with respect to the emergency department arrival time. FIRST, the Fast Interrogation Rule for ST-segment elevation myocardial infarction program. HOD, hospital day.

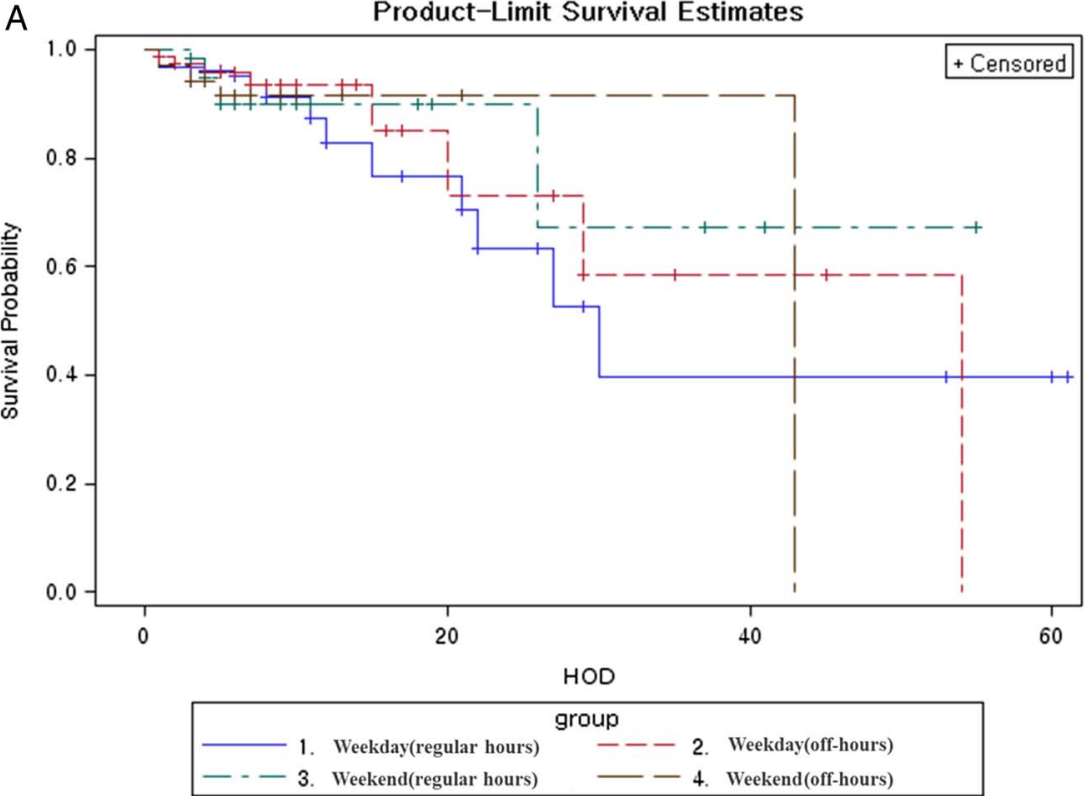

B

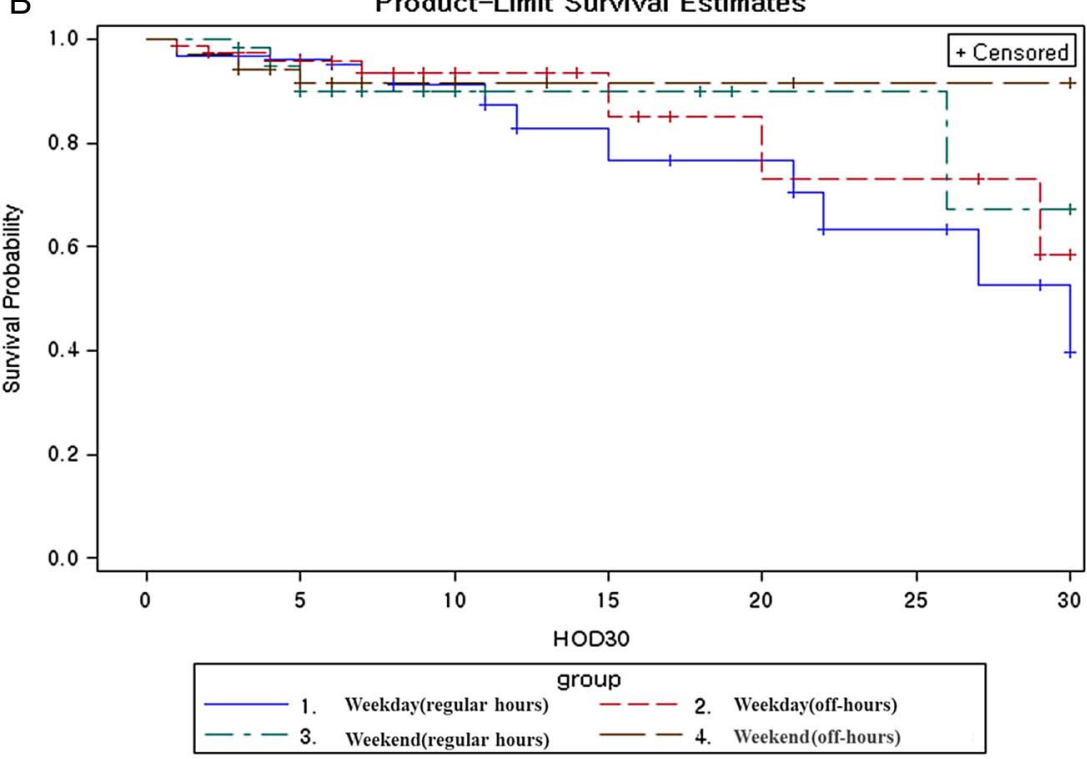

The low level of awareness of the public and EMS in Korea may lead to the importance of rapid recognition, diagnosis and treatment of ACS and cardiac arrest being neglected. Frequently, patients with STEMI do not exactly describe symptoms characteristic of STEMI. Despite risk stratification in the ED, it is thus possible that cardiologists might aggressively conduct primary PCI. Several studies have reported that the proportion of patients with AMI with door-to-balloon times $<90 \mathrm{~min}$ varies, ranging from $6.3 \%$ to $98.5 \% .{ }^{14}{ }^{20} \mathrm{Pan}$ et a ${ }^{9}$ showed that implementing specific strategies improved the proportion of patients who underwent PCI within 90 min from $59.4 \%$ to $98.5 \%(p<0.001)$. On the other hand, our study did not show significant differences in the proportions of patients who underwent PCI within 90 min, regardless of the arrival time at the $\mathrm{ED}$, ranging from $89.6 \%$ to $95.1 \%$. Several studies have, moreover, reported that there was no significant difference in mortality in patients with AMI admitted during regular versus off-hours. ${ }^{19} 2122$ However, in one study, patients with AMI admitted during off-hours were less likely to undergo primary PCI and more likely to undergo fibrinolytic therapy. Consequently, there was no difference in the overall usage of reperfusion therapy, irrespective of the arrival time. ${ }^{21}$ In another study, the clinical effectiveness of PCI performed in a highly specialised STEMI network system was equivalent between regular and off-hours admissions at the regional level. ${ }^{22}$ A dedicated cardiac care network usually consists of a prehospital pathway, EMS training, priority for laboratory studies and emergency care and 24 hours/day and 7 days/week availability of specialised cardiologists. ${ }^{10}$ The coordination of the above elements can overcome the deleterious effects of admission during off-hours 
and weekends. ${ }^{10}$ However, it should be noted that many of the previous studies on the topic were conducted in areas with well-organised cardiac care centres in developed countries.

Practice guidelines for decision support and diseasespecific order sets can be embedded in the CPOE system. ${ }^{9} 1{ }^{103}$ In clinical practice, a CPOE system based on predetermined order sets is not simply a computer network, but can provide many communication advantages, including clarification and tracking of the completion of orders and errors. ${ }^{9}$ Our FIRST protocol, based on a CPOE system, facilitates the simultaneous usage of a multidisciplinary team approach, which has benefits for reducing medical errors and improving the quality of the critical process. ${ }^{10}$ However, the successful operation of a CP requires standardisation and simple protocols, regular educational opportunities and feedback from clinical results. ${ }^{10}$ As in the story of 'crying wolf' in Aesop's fables, repeated false and true alarms may cause an alarm fatigue effect and desensitisation to alarms. ${ }^{24}$ In patients with ischaemic stroke, it has been reported that the behaviour of the physician may result in a so-called 3-hour effect and a longer door-to-needle waiting time. ${ }^{12}$ Nevertheless, alarm fatigue can be reduced and the behaviour of physicians can be changed by using a multidisciplinary team approach and education on the relevant issues, the optimisation of alarms and a systematic perspective for reducing alarm fatigue. $^{24} 25$

This study should be considered in the context of several limitations. First, it was impossible to perform a direct internal comparison with clinical data obtained before the implementation of a CP based on the CPOE system, because it was difficult to select a cohort with inclusion criteria similar to our study population. Further studies are needed to assess the outcomes before and after the implementation of a CP based on CPOE for patients with AMI. Second, we only assessed short-term mortality and time factors in a relatively small population during a 30-day period and a single LOS. In the future, prospective multicentre studies with a larger number of patients will be needed to confirm the usefulness of implementation of this CP in terms of the time factors and mortality, including long-term outcomes, in patients with STEMI. Finally, delayed diagnosis of STEMI and the presence of severe disease can increase the door-to-balloon time and mortality; however, patients who experienced return of spontaneous circulation after out-of-hospital cardiac arrest or ST-segment elevation on follow-up ECG only were not included in this study, because it would have been impossible to compare the time factors based on the arrival time at the ED for these patients.

\section{CONCLUSION}

A multidisciplinary CP based on a CPOE system for STEMI can effectively decrease disparities in the door-to-data interval and the proportions of patients with door-to-balloon times within $90 \mathrm{~min}$, regardless of the ED arrival time. The application of a multidisciplinary CP may also help attenuate off-hour and weekend effects in the clinical outcomes of patients with STEMI.

Author affiliations

${ }^{1}$ Department of Emergency Medicine, Yonsei University College of Medicine, Seoul, Republic of Korea

${ }^{2}$ Department of Research Affairs, Biostatistics Collaboration Unit, Yonsei University College of Medicine, Seoul, Republic of Korea

Contributors YSP, SPC, HSL, JHH and JSY conceived and designed the study. YSP, MJK, HSC, SPC, JW, IP and JSY performed the study, YSP, SPC HSL, JHH, IP and JSY analysed the data. YSP, JSY and SPC contributed to the writing of the manuscript. All authors contributed in editing of the manuscript.

Funding This work was supported by the Basic Science Research Program through the National Research Foundation of Korea funded by the Ministry of Education (grant number NRF-2013R1A1A2009391), and by the Ministry of Science, ICT and Future Planning (grant number

NRF-2015R1C1A1A01054641).

Competing interests None declared.

Ethics approval The institutional review board of Yonsei University Health System (Number 3-2015-056).

Provenance and peer review Not commissioned; externally peer reviewed.

Data sharing statement No additional data are available.

Open Access This is an Open Access article distributed in accordance with the Creative Commons Attribution Non Commercial (CC BY-NC 4.0) license, which permits others to distribute, remix, adapt, build upon this work noncommercially, and license their derivative works on different terms, provided the original work is properly cited and the use is non-commercial. See: http:// creativecommons.org/licenses/by-nc/4.0/

\section{REFERENCES}

1. Bell CM, Redelmeier DA. Mortality among patients admitted to hospitals on weekends as compared with weekdays. $N$ Engl J Med 2001;345:663-8.

2. Sorita A, Ahmed A, Starr SR, et al. Off-hour presentation and outcomes in patients with acute myocardial infarction: systematic review and meta-analysis. BMJ 2014:348:f7393.

3. O'Connor RE, Bossaert L, Arntz HR, et al. Part 9: acute coronary syndromes: 2010 International consensus on cardiopulmonary resuscitation and emergency cardiovascular care science with treatment recommendations. Circulation 2010;122:S422-65.

4. Mozaffarian D, Benjamin EJ, Go AS, et al. Heart disease and stroke statistics-2015 update: a report from the American Heart Association. Circulation 2015;131:e29-322.

5. O'Gara PT, Kushner FG, Ascheim DD, et al. 2013 ACCF/AHA guideline for the management of ST-elevation myocardial infarction: executive summary: a report of the American College of Cardiology Foundation/American Heart Association Task Force on Practice Guidelines: developed in collaboration with the American College of Emergency Physicians and Society for Cardiovascular Angiography and Interventions. Catheter Cardiovasc Interv 2013;82:E1-27.

6. Levine GN, Bates ER, Blankenship JC, et al. 2015 ACC/AHA/SCAI Focused Update on primary percutaneous coronary intervention for patients With ST-Elevation Myocardial Infarction: an Update of the 2011 ACCF/AHA/SCAI Guideline for Percutaneous Coronary Intervention and the 2013 ACCF/AHA Guideline for the Management of ST-Elevation Myocardial Infarction: a report of the American College of Cardiology/American Heart Association Task Force on Clinical Practice Guidelines and the Society for Cardiovascular Angiography and Interventions. Circulation 2016;133:1135-47.

7. Bajaj S, Parikh R, Gupta N, et al. "Code STEMl" protocol helps in achieving reduced door-to-balloon times in patients presenting with acute ST-segment elevation myocardial infarction during off-hours. J Emerg Med 2012;42:260-6. 
8. Kostis WJ, Demissie K, Marcella SW, et al. Weekend versus weekday admission and mortality from myocardial infarction. $N$ Engl J Med 2007;356:1099-109.

9. Pan MW, Chen SY, Chen CC, et al. Implementation of multiple strategies for improved door-to-balloon time in patients with ST-segment elevation myocardial infarction. Heart Vessels 2014;29:142-8.

10. Yang JM, Park YS, Chung SP, et al. Implementation of a clinical pathway based on a computerized physician order entry system for ischemic stroke attenuates off-hour and weekend effects in the ED. Am J Emerg Med 2014;32:884-9.

11. Ryu DR, Choi JW, Lee BK, et al. Effects of critical pathway on the management of patients with ST-elevation acute myocardial infarction in an emergency department. Crit Pathw Cardiol 2015;14:31-5.

12. Heo JH, Kim YD, Nam HS, et al. A computerized in-hospital alert system for thrombolysis in acute stroke. Stroke 2010;41:1978-83.

13. Cho HY, Na S, Kim MD, et al. Implementation of a multidisciplinary clinical pathway for the management of postpartum hemorrhage: a retrospective study. Int J Qual Health Care 2015;27:459-65.

14. Abi Rafeh N, Abi-Fadel D, Wetz RV, et al. A STEMI code protocol improves door-to-balloon time on weekdays and weekends. J Healthc Qual 2009;31:35-43.

15. Holmes DR Jr, Bell MR, Gersh BJ, et al. Systems of care to improve timeliness of reperfusion therapy for ST-segment elevation myocardial infarction during off hours: the Mayo Clinic STEMI protocol. JACC Cardiovasc Interv 2008;1:88-96.

16. Al-Asadi JN, Kadhim FN. Day of admission and risk of myocardial infarction mortality in a cardiac care unit in Basrah, Iraq. Niger J Clin Pract 2014;17:579-84.
17. Ostrzycki A, Sosnowski C, Borowiec-Kocańda A, et al. Pre-hospital delay of treatment in patients with ST segment elevation myocardial infarction undergoing primary percutaneous coronary intervention: experience of cardiac centre located in the vicinity of the centre of Warsaw. Kardiol Pol 2008;66:609-14; discussion 15-6.

18. Chughtai H, Ratner D, Pozo M, et al. Prehospital delay and its impact on time to treatment in ST-elevation myocardial infarction. Am J Emerg Med 2011;29:396-400.

19. Na JP, Shin KC, Kim S, et al. Performance of reperfusion therapy and hospital mortality in ST-elevation myocardial infarction patients with non-chest pain complaints. Yonsei Med J 2014;55:617-24.

20. Magid DJ, Wang Y, Herrin J, et al. Relationship between time of day, day of week, timeliness of reperfusion, and in-hospital mortality for patients with acute ST-segment elevation myocardial infarction. JAMA 2005;294:803-12.

21. Jneid $\mathrm{H}$, Fonarow $\mathrm{GC}$, Cannon $\mathrm{CP}$, et al. Impact of time of presentation on the care and outcomes of acute myocardial infarction. Circulation 2008;117:2502-9.

22. Casella G, Ottani F, Ortolani P, et al. Off-hour primary percutaneous coronary angioplasty does not affect outcome of patients with ST-segment elevation acute myocardial infarction treated within regional network for reperfusion: The REAL (Registro Regionale Angioplastiche dell'Emilia-Romagna) registry. JACC Cardiovasc Interv 2011;4:270-8.

23. Nam HS, Han SW, Ahn SH, et al. Improved time intervals by implementation of computerized physician order entry-based stroke team approach. Cerebrovasc Dis 2007;23:289-93.

24. Sendelbach S, Funk M. Alarm fatigue: a patient safety concern. AACN Adv Crit Care 2013;24:378-86; quiz 87-8.

25. Blake N. The effect of alarm fatigue on the work environment. AACN Adv Crit Care 2014:25:18-19. 